\title{
Imaging Frontiers
}

\section{Potential solutions for confocal imaging of living animals}

\author{
Claudette St. Croix ${ }^{1}$, Warren R. Zipfel2, and Simon C. Watkins ${ }^{1}$
}

BioTechniques 43:S14-S19 (July 2007)

doi 10.2144/000112509

\begin{abstract}
The use of confocal and multiphoton microscopy for in vivo studies in animals continues to be an area of exciting technical and commercial development. However, the application of these technologies at high resolution, such that molecular and subcellular information is collected, remains an elusive goal. This review discusses the practical and performance limitations and the potential uses of currently available systems. We also highlight the ongoing developments in both miniaturized and bench-mounted systems for single and multiphoton optical sectioning studies in animals and in human clinical trials.
\end{abstract}

\section{POINT-SCANNING CONFOCAL AND MULTIPHOTON APPROACHES}

Confocal microscopy has become a centrally important tool to the research microscopist. The technology allows optical sectioning of cells and tissues and recovery of focused, low noise images. The basic principal behind confocal microscopy is to deliver illumination and collect the returned signal through a pinhole of defined size that rejects out-of-focus light. Clearly however, this simple system will only collect light from a single point in the specimen. To collect a full image of the specimen, it is necessary to move the point source relative to the specimen. In fact, in the first usable design, developed in the late 1950s (1), the pinhole and illumination stayed stationary, and the specimen was scanned beneath the pinhole using a scanning stage. However, this approach is extremely slow (due to the mass of the stage) and not a currently useful solution. The first practical application (2) of the technology we use today was introduced 20 years ago. This instrument, which as a commercial device became the Bio-Rad MRC
500, moved the illumination relative to the specimen using two galvanometer-driven mirrors (one scanning rapidly, at approximately 1000 times/s in one axis, and the other scanning slowly, at $1 / \mathrm{s}$ in the orthogonal axis). This raster-based approach built a two-dimensional image in a similar fashion to a simple television image. Since this seminal development, there have been innumerable reinventions of the same conceptual design, some using a single mirror scanning in both axes, others a combination of stepping motors and galvonometers. All devices were relatively slow and, apart from the devices made by the "big four" microscope companies (Leica, Olympus, Nikon, and Zeiss), none have survived to the current day.

Concurrently, there has been a constant need to develop machines that could work faster with high signalto-noise ratios and with multiple fluorescent markers. This need was principally driven by physiologists, who required the ability to examine ionic reporters in real-time in living systems. Scanheads that were developed in these early days were extraordinary in complexity, but were fundamentally limited by the sensitivity of the detectors. A greater problem, however, was the performance limitations in the computers of the time, which were challenged both from the point of view of data throughput and storage.

Today, the majority of the problems that limited the use of conventional bench-mounted confocal microscopy have been resolved, and while point-scanning laser-based confocal microscopes are only sold by the four major microscope companies, all the available systems are extremely sensitive, flexible, and have highly sophisticated and useful analysis software. Furthermore, high-speed confocal imaging is becoming much more practical, with the ability to collect images at frame rates of over $100 \mathrm{frames} / \mathrm{s}$, at high resolution, and with multiple colors. These systems either work in line scan mode rather than point scan or use multiple focal volumes that are rapidly scanned across the sample. More recently, these latter technologies have been facilitated by major developments in electron multiplier array technologies that have much higher sensitivity than cameras available even 5 years ago.

The investigator, however, continues to demand more flexibility 


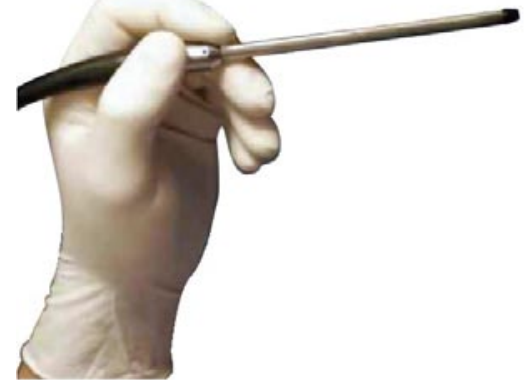

Figure 1. OptiScan FIVE 1 confocal microscope scanhead. Image courtesy of Optiscan Pty. Ltd. (Victoria, Australia).

and utility from the confocal microscope. In particular, there is a constant need to image deeper into tissues or to image specimens that do not conform to the constraints of a conventional microscope platform. Deep tissue imaging in living animals has, to some degree, been solved using multiphoton approaches. This technology, developed at Cornell in 1990 (3), uses ultrafast pulsed long wavelength lasers as the illumination source. If two long wavelength photons arrive at a fluorophore essentially simultaneously, they act synergistically, donating the photonic energy of a shorter wavelength photon, and hence exciting the fluorophore. One reason this approach allows deeper penetration is that long wavelength light is absorbed and scattered much less than short wavelength light. Furthermore, as fluorophore excitation only occurs at the focal plane of the lens, no pinhole is needed on the emission side of the device. As such, even if the emission light is scattered dramatically by the tissue, it can still reach the detector and provide useful signal since precise focusing of the light through a pinhole is not required. However, penetration is still limited to a few hundred microns and generally to samples that can be placed conveniently on a conventional microscope stage.

\section{IN VIVO IMAGING NEEDS}

There is a considerable need to develop imaging platforms that have the flexibility and sensitivity of modern confocal and multiphoton imaging systems, but which can be used in less orthodox ways, such that they are applicable to imaging problems that cannot be constrained by the benchtop microscope. Examples are: (i) imaging within culture systems and bioengineered model organ systems that do not fit within a microscope (e.g., small aperture transwell culture dish or large-scale tissue engineering factories for skin or liver); (ii) imaging of irregular surfaces, such as in the knee for example or in situ on production lines; and (iii) imaging in animal model systems (both mice and larger animals, such as sheep and nonhuman primates) or, as an ultimate goal, within humans in clinical trials. The currently available systems, which are finding real utility in the realm of the experimental scientist, have grown from clinical devices and make use of all the optical and miniaturization developments made for this much larger market.

Historically, there have been several competing confocal and nonconfocal imaging methods developed with the ultimate goal of in vivo imaging. Optical coherence tomography (OCT), which is able to produce an $\mathrm{x} / \mathrm{z}$ slice through the tissue in a noninvasive manner, has been investigated extensively as a topical diagnostic tool for the dermatologist and other medical specialties such as ophthalmology. OCT can be fiber-based using an extremely small probe (essentially the same size as an optical fiber) to generate a section in the $\mathrm{z}$ plane (similar to a conventional histologic section) using backscattered light from endogenous tissue structures. Developing refinements of this technology is currently an area of intensive research (4), however there are two primary limitations. First, the resolution is at best cellular, and secondly and perhaps more importantly, the approach is fundamentally only descriptive as the signal is dependent on endogenous structure and does not provide molecular information (although some new forms, such as molecular contrast OCT, do address this problem). Ongoing improvements in OCT continue to occur, but the approach has yet to find significant utility in experimental biology or medicine.

\section{FIBER-BASED CONFOCAL APPROACHES}

Novel confocal approaches that deviate from the standard microscope stand/laser scanner configuration can be divided into fiber bundle-based systems and single fiber-based systems in which a single fiber is used for excitation delivery and, in some cases, delivery of the emission back through the fiber to a detector. The fiber bundlebased systems are extremely smallessentially a bundle of fibers, $1-2 \mathrm{~mm}$ in diameter, that can contain upwards of 30,000 fibers. Each fiber acts as a light delivery and collection system and is, in essence, its own pinhole. This type of system can be considered equivalent to an insect eye, each fiber acting as an independent omatidi. The absolute advantages of this design are size, flexibility, and image collection speed. As all the light delivery, collection, filtering, and imaging is at the proximal end of the fiber bundle, the distal end of the device is simply a lens and the fiber bundle. This means that the whole system is extremely flexible and extremely small. Frame rates of $12 \mathrm{frames} / \mathrm{s}$ are possible, considerably faster than any of the conventional high-resolution point scanners, and help avoid artifacts due to specimen movement. On the other hand, the image is pixel-limited, and even with a bundle of 30,000 fibers, the final image is on the order of $200 \times 200$ resolvable elements (or resels), which is less than one quarter of a typical $512 \times 512$ image and 1/20th of a typical high-resolution point-scanned image. Furthermore, the individual fibers within the bundle are separate and, even if perfectly packed, result in a loss of signal in the fiber bundle due to cladding around each light guiding element. Lastly, the focus point of the bundle is fixed, so that $\mathrm{z}$ series typical of confocal microscopy are not possible.

The current commercial device is called the Cellvizio ${ }^{\circledR}$ (Mauna Kea Technologies, Cambridge MA, USA). Its very small diameter means that it can be used within the operations channel of a current clinical endoscope. Thus it is an exceptionally flexible device, compatible with currently available endoscopy without the need to build 


\section{Imaging Frontiers}

or procure novel endoscopy tools. A laboratory version is also available, and the small diameter of the probe and the flexibility of the fiber optic fiber allows long-term insertion into animals such that chronic processes can be followed. This device has been used to image various neural structures in the living animal $(5,6)$ and the microstructure of the bronchial wall (7). One important caveat, however, is that the thinner the physical diameter of the fiber bundle, the lower the number of fibers in the bundle, and hence the lower the effective pixel count, ultimately limiting the resolution. Thus with the thinnest fiber available, the reported resolution is $3.5 \mu \mathrm{m}$. This is just at the limits of subcellular resolution (e.g., a lymphocyte is approximately $7 \mu \mathrm{m}$ in diameter). On the other hand, the larger fiber bundles have lateral resolutions of $<2 \mu \mathrm{m}$. In summary, the fiber bundle approach is certainly the thinnest and most physically flexible system currently available and can be used for applications in which other rigid probe systems are simply unworkable. However, these advantages come with the drawback that resolution is ultimately limited by the number of fibers in the bundle.

A second potential approach is to use microelectromechanical system (MEMS) mirrors to build a microscope in which the scanhead is micromachined, such that it could be of submillimeter physical size $(8,9)$. There is considerable potential using this approach, and although at this time no commercially available instruments have been developed, several groups have built prototype MEMSbased miniature scanners. For example, the Wang group at Stanford has recently demonstrated a unique dual-axes near infrared (NIR) confocal microendoscope (10) with an outer diameter of $10 \mathrm{~mm}$ that can be used for subsurface imaging of biological tissues. The device is capable of acquiring images at $30 \mathrm{frames} / \mathrm{s}$, over a field of view of $800 \times 100 \mu \mathrm{m}$, with a resolution of 5-7 $\mu \mathrm{m}$. It uses a novel two-dimensional MEMS mirror scanner and can obtain both reflectance and NIR fluorescence images using a laser source at $785 \mathrm{~nm}$. This and other similar developments predict usable commercial systems within the next few years.
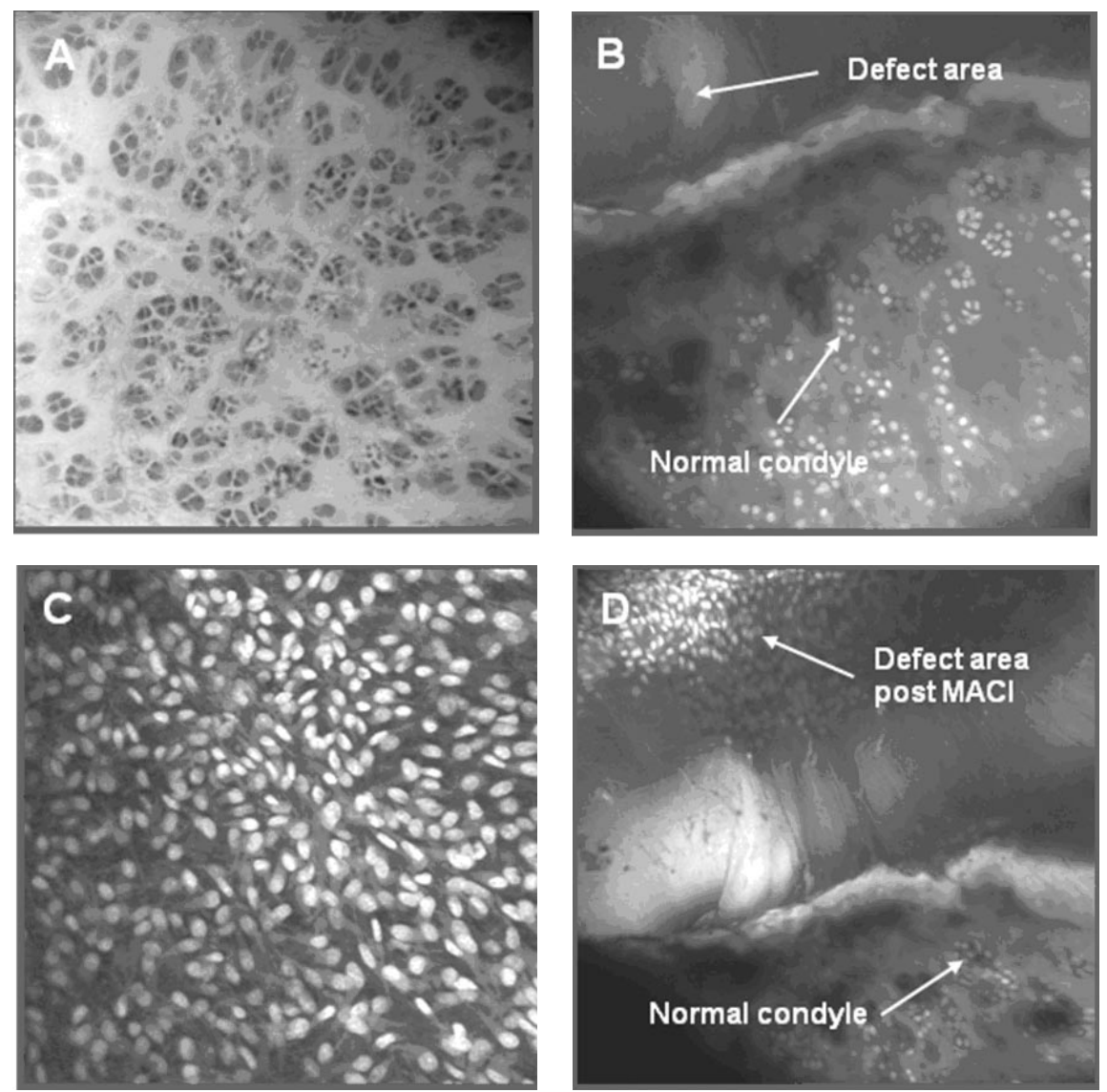

Figure 2. Early osteoarthritic changes and chondrocyte transplant success in sheep. (A) Noninvasive in vivo imaging of chondrocytes and lacunae in articular cartilage. (B) In a sheep model of cartilage damage, dramatic changes are observed at the site of tissue injury. (C) Following matrixinduced autologous chondrocyte implantation (MACI), there is a dramatic increase in the number of chondrocytes. (D) Assessement of the efficacy of cartilage repair techniques such as MACI. Image courtesy of Drs. Chris Jones and Brett Kirk, University of Western Australia, Perth, Western Australia.

\section{SINGLE FIBER-BASED CONFOCAL APPROACHES}

Solutions that have found much more promise over the last several years and are now commercially available combine conventional confocal components with novel miniaturization strategies, such that scan resolutions and pixel counts similar to the standard bench confocal have been achieved. Historically, the systems that have come to a current commercial reality were originally developed for diagnostic purposes, specifically in dermatology. The first, the VivaScope ${ }^{\circledR}$, developed by Lucid Technologies (Rochester, NY, USA) is a NIR reflectance mode confocal microscope developed for dermatologic investigations. The original device (VivaScope 1500) uses essentially a standard microscope objective lens and a large scanhead coupled to a rigid but flexible mount with three-axis motion. Reflectance mode confocal microscopy does not rely on fluorescence, but rather interrogates tissue by illuminating with a long wavelength continuous wave laser and collecting reflected light (sorted from polarized exciting light with a quarter waveplate) to form the image. The advantages of this approach are deep penetration (due to the long wavelength illumination) and a fast frame rate (achieved using a rotating polygonal mirror). The disadvantage is that no true molecular information is available, in that only structural images are formed based on tissue differences that alter the amount of backscattered light (similar to OCT above). The VivaScope 1500 is also a relatively large device and not 


\section{Imaging Frontiers}
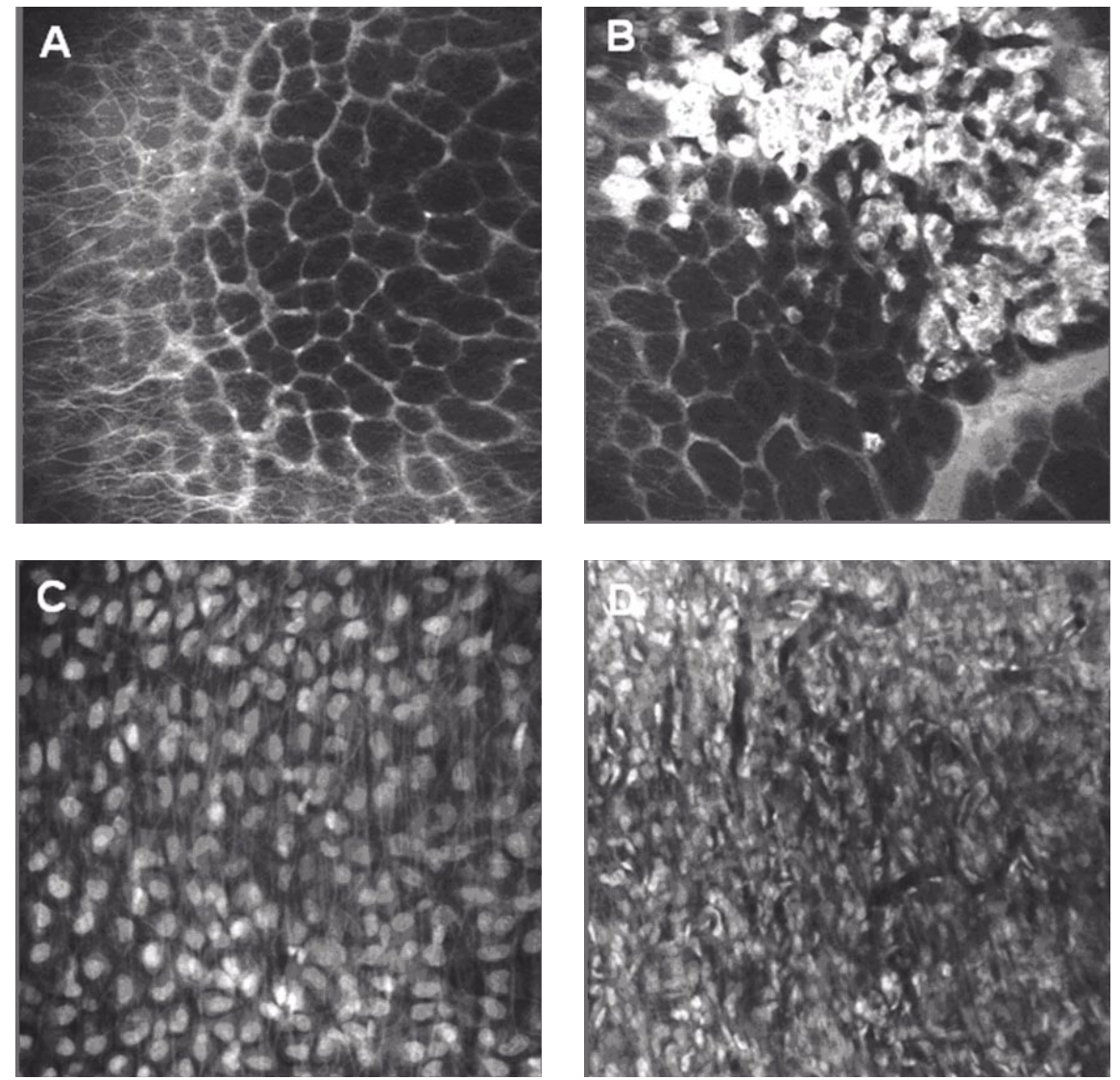

Figure 3. Noninvasive microscopy enabling longitudinal studies of molecular and microvascular events in a rat model of pancreatic cancer. (A) Noninvasive in vivo imaging of normal pancreatic microvasculature. (B) Molecular staining of rat pancreatic islet cells can be imaged by targeting the somatostatin receptor with 5-carboxyfluorescein-labeled ocreotate. (C) Fluorescent staining of rat body wall shows a confluent layer of healthy cells. (D) Visualization of body wall metastases in a rat model of pancreatic cancer. Image courtesy of Drs. Martin Goetz and Ralf Kiesslich, University of Mainz, Mainz, Germany.

readily usable as an intravital scanner. However, Lucid Technologies has recently introduced a smaller handheld version (VivaScope 3000). Although the targeted users are dermatologists, this smaller device could be used for in vivo experimental confocal imaging in animal model systems in which fluorescence is not required.

The second system, originally developed by Optiscan (Notting Hill, Melbourne, Australia) also used conventional optics, but was a fluorescencebased confocal microscope rather than a reflection mode confocal. This device was also quite large and, like the VivaScope 1500 , was also developed for the dermatology market. Both of these products found resistance from the dermatopathology market for various reasons; they were complex to use, cumbersome, and limited in their application.

To bring these technologies to real utility for the bench scientist, it was necessary to combine the miniaturized aspects of the Mauna Kea head, with the high-resolution scanning mechaconfocal. Perhaps the biggest limitation in the prior designs was the need to use standard, commercially available lenses. None of the companies attempting to build miniature confocal microscopes for in vivo use had the fiscal resources to develop truly miniature high numeric aperture (NA) objective lenses. Although several research groups have built miniaturized confocal and multiphoton endomicroscopes that utilize a gradient index (GRIN) lens as the objective lens $(11,12)$, the optical performance of nisms possible with a conventional these systems has been relatively poor compared with a well-designed microscope objective of the same NA. More recently, there have been major developments in the design and fabrication of high-quality miniaturized objective lenses. Optiscan has worked closely with Pentax to build a confocal colonoscope that is now commercially available (since 2006). As part of this design, it was necessary to integrate the Optiscan fiberscanning technologies with a novel lens design. Ultimately, the companies codeveloped an extremely small scanhead ( $4.5 \mathrm{~cm}$ long $\times 3.5 \mathrm{~mm}$ diameter), which has been used in published clinical trials (13-17), and have started to validate the optical biopsy as a rational clinical solution for endoscopy.

An offshoot of this technology has been a new design for handheld rigid confocal microscopy, which essentially combines the technologies developed for confocal colonoscopy with a rigid handheld device. The current device is shown in Figure 1 and is currently allowing us to image a variety of in situ reporters in living animals, which was not possible using a bench-mounted confocal. Applications of this device fall broadly into three categories: $(i)$ morphological studies; (ii) cell tracking studies, whereby specific cell populations can be fluorescently labeled and subsequently tracked within whole animal models; and (iii) molecular studies, whereby fluorescent reporters allow imaging of specific protein expression in vivo. In Figure 2, noninvasive imaging enabled detection of early osteoarthritic changes and the longitudinal study of chondrocyte transplant success in sheep. In this example, sheep knee ligaments were surgically operated to create knee misalignment. Following recovery from surgery, sheep were walked on a treadmill to create damage to the joint. Changes in the cellular morphology of the knee cartilage (trochlear groove of the femur) were examined following the topical application of fluorescein sodium (Figure $2 \mathrm{~A}$ ) or acriflavine hydrochloride (Figure 2, B-D). Chondrocytes contained within small lacunae of articular cartilage could be clearly observed. Matrix-induced autologous chondrocyte implantation 


\section{Imaging Frontiers}
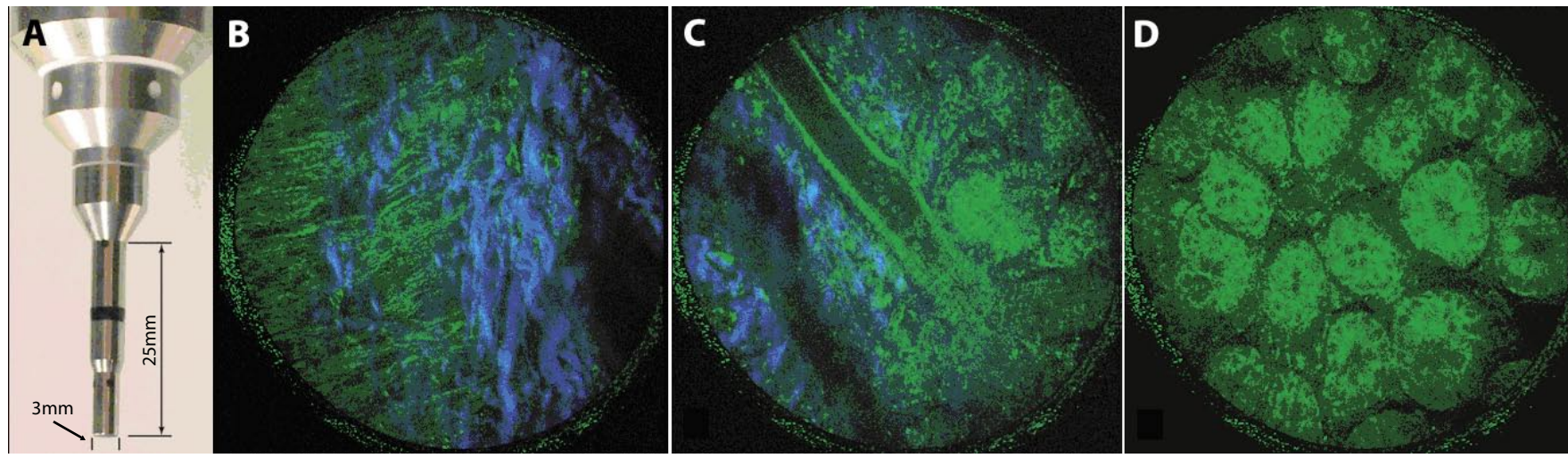

Figure 4. In vivo multiphoton imaging through a stick objective. (A) $27 \times / 0.7$ NA Olympus stick objective. (B) The outer surface of a mouse small bowel. (C) Approximately $150 \mu \mathrm{m}$ below the outer surface. (D) The base of villi observed through the outer bowel wall. Excitation at $760 \mathrm{~nm}$ (approximately $150 \mathrm{fs}$ pulse width); green pseudocolor, 400-500 nm intrinsic fluorescence; blue pseudocolor, collagen second-harmonic signal (380 nm).

(MACI) was performed to repair defects in the articular cartilage.

The second example (Figure 3) illustrates how noninvasive microscopy enables longitudinal studies of molecular and microvascular events in a rat model of pancreatic cancer (18) via imaging of somatostatin receptor positive cells (expressed on most gastroenteropancreatic neuroendocrine tumors). Figure $3 \mathrm{~A}$ shows the healthy pancreatic microvasculature of the rat. Pancreatic islet cells were stained by targeting somatostatin receptor subtype 2 (sstr2) with 5-carboxyfluoresceinlabeled octreotate (OcF) (Figure 3B). Cells of the healthy body wall were examined in experimental rats through a small abdominal incision (Figure 3C). The incision was then sutured closed, and the rats were allowed to recover from surgery to enable the development of body wall metastases (Figure 3D).

Olympus has also produced a small fiber delivered confocal scanner (IV-100) designed specifically for in vivo experimental research. Key to this device was the design of "stick" objective lenses that range from 3.2 to $1.3 \mathrm{~mm}$ in diameter with a probe length of approximately $1 \mathrm{~cm}$ (see Figure 4). The three objectives currently available have NAs of 0.7 (3.2 $\mathrm{mm}$ in diameter), 0.5 , and 0.14 (both $1.3 \mathrm{~mm}$ in diameter), and $220 \mu \mathrm{m}(27 \times), 200 \mu \mathrm{m}(20 \times)$, and $670 \mu \mathrm{m}(6 \times)$ fields of view, respectively. The complete IV-100 system has three detectors, multiple laser lines, and the small scanhead is mounted on a threeaxis articulated arm so the head can be positioned at any angle required during imaging.

\section{FUTURE DEVELOPMENTS}

In the area of laser-scanning microscopy, multiphoton approaches have greatly advanced imaging of thick samples such as tissue explants and in vivo imaging in small animals (19). The extension of nonlinear excitation to the types of novel laser scanners discussed above will further enable the direct visualization of intracellular events in the intact tissue using whole animal model systems and potentially in human clinical applications. Multiphoton excitation not only allows for deeper imaging than possible using single-photon excitation, but permits the simultaneous excitation of multiple fluorophores with a single laser, as well as direct imaging of intrinsic tissue signals such as cellular and extracellular autofluorescence and secondharmonic generation signals for tissue constituents such as collagen. This latter attribute should prove to be particularly important in clinical applications in which exogenous labels cannot be added. There are complications that arise when applying multiphoton excitation to microendoscopic applications (in addition to those already discussed). Efficient two-photon excitation requires the shortest possible laser pulsewidth to minimize the average power and maximize the pulse peak energy. This becomes a serious problem when trying to deliver short pulses through optical fibers, which are essentially long lengths of dispersive glass. Ultrashort pulses of light are not a single wavelength, but typically a limited collection of colors around the peak wavelength. For example, when the Ti:sapphire laser of a multiphoton microscope is tuned to $800 \mathrm{~nm}$, the most intense wavelength is $800 \mathrm{~nm}$, but this is the peak of a Gaussian distribution centered at $800 \mathrm{~nm}$ with a full width at half maxium of 10-15 nm. Glass causes a dispersion in which the shorter wavelengths lag behind the longer wavelengths as the pulse travels through the fiber that broadens the pulsewidth. Typical 100 -fs pulses passing through $1 \mathrm{~m}$ of conventional optical fiber are stretched 10-fold resulting in an approximately 4-fold decrease in excitation efficiency. Although some multiphoton microscopes use pulse compensation schemes to correct for the microscope optics, this is not totally effective. However, fiber-delivered multiphoton microendoscopy has been accomplished using conventional single-mode fibers with the resulting longer pulsewidths (20), but new fiber designs are now becoming available that alleviate the problem and allow for efficient multiphoton excitation delivery through a fiber. These new fibers either allow a specific narrow wavelength region to pass through the fiber without any dispersion (21), or the degree of noncompensatible nonlinear dispersion is rendered negligible. This can be achieved using large core (multimode) microstructured fibers (22) that are still capable of delivering a singlemode profile (i.e., a single Gaussian profile spot out of the fiber) of pulsed laser light. By applying a standard pulse compensation scheme (typically a prism or grating pair), approximately 100 -fs pulses can be achieved at the 
focal point. Equally exciting are developments in the design and fabrication of higher order mode fibers (23) that will enable both short pulse delivery from existing laser sources as well provide a basis for new designs of inexpensive femtosecond fiber lasers.

A final aspect that will greatly improve this type of minimally invasive in vivo imaging, both when using multiphoton excitation as well as for single-photon excited confocal microendoscopy, is the increasing availability of new high-sensitivity photomultiplier tubes (PMTs). GaAsP photocathode devices such as the Hamamatsu H7422P-40 have greater than $40 \%$ photocathode quantum efficiency in the approximately 500-600 $\mathrm{nm}$ region; almost twice that of current generically used PMTs. More recently, detection in the UV and blue region of the spectrum was significantly enhanced with the introduction of the new ultra-bialkali (UBA) photocathode, also from Hamamatsu Corporation (Bridgewater, NJ, USA). Given that the numerical aperture of microendoscopic systems will remain appreciably less than that available in a standard laser-scanning microscope, increasing detector efficiency can partially compensate for this loss in collection efficiency.

Currently available components can be used to modify existing multiphoton microscopes to improve in vivo imaging of animal models. For example, the Olympus stick objective (discussed above) works well using multiphoton excitation. An example of using the stick lenses as a miniature multiphoton laparoscope is shown in Figure 4. While these images were taken with the objective mounted on a conventional upright microscope, rather than one of the previously discussed miniature fiber-delivered scanners, it does demonstrate that it is possible to image through the intestinal wall of a mouse using only intrinsic fluorescence and collagen second-harmonic generation. These are typically weak signals compared with the fluorophores and fluorescent proteins commonly used in research, and an optimally designed multiphoton microendoscope system should greatly enhance the imaging depth possible for typical intravital research projects.

\section{ACKNOWLEDGMENTS}

This was work was supported by National Institutes of Health (NIH) grant nos. 7U54RR022241-02 (to S.C.W.), HL-081421 (to C.M.S.), and CA116583 (to W.R.Z.).

\section{REFERENCES}

1. Minsky, M. 1988. Memoir on inventing the confocal scanning microscope. Scanning 10:128-138.

2. White, J.G., W.B. Amos, and M. Fordham. 1987. An evaluation of confocal versus convential imaging of biological structures by fluorescence light microscopy. J. Cell Biol. 105:41-48.

3. Denk, W., J.H. Strickler, and W.W. Webb. 1990. Two-photon laser scanning fluorescence microscopy. Science 248:73-76.

4. Yaqoob, Z., J. Wu, E.J. McDowell, X. Heng, and C. Yang. 2006. Methods and application areas of endoscopic optical coherence tomography. J. Biomed. Opt. 11:063001.

5. Bharali, D.J., I. Klejbor, E.K. Stachowiak, P. Dutta, I. Roy, N. Kaur, E.J. Bergey, P.N. Prasad, and M.K. Stachowiak. 2005. Organically modified silica nanoparticles: a nonviral vector for in vivo gene delivery and expression in the brain. Proc. Natl. Acad. Sci. USA 102:11539-11544.

6. Vincent, P., U. Maskos, I. Charvet, L. Bourgeais, L. Stoppini, N. Leresche, J.P. Changeux, R. Lambert, et al. 2006. Live imaging of neural structure and function by fibred fluorescence microscopy EMBO Rep. 7:11541161.

7. Thiberville, L., S. Moreno-Swirc, T. Vercauteren, E. Peltier, C. Cave, and G. Bourg Heckly. 2007. In vivo imaging of the bronchial wall microstructure using fibered confocal fluorescence microscopy. Am. J. Respir. Crit. Care Med. 175:22-31.

8.Flusberg, B.A., E.D. Cocker, W. Piyawattanametha, J.C. Jung, E.L.M. Cheung, and M.J. Schnitzer. 2005. Fiber-optic fluorescence imaging. Nat. Methods 2:941950.

9. Flusberg, B.A., J.C. Jung, E.D. Cocker, E.P. Anderson, and M.J. Schnitzer. 2005. In vivo brain imaging using a portable 3.9 gram twophoton fluorescence microendoscope. Opt. Lett. 30:2272-2274

10.Liu, J.T, M.J. Mandella, H. Ra, L.K. Wong, O. Solgaard, G.S. Kino, W. Piyawattanametha, C.H. Contag, and T.D. Wang. 2007. Miniature near-infrared dualaxes confocal microscope utilizing a twodimensional microelectromechanical systems scanner. Opt. Lett. 32:256-258.

11.Jung, J.C., A.D. Mehta, E. Aksay, R. Stepnoski, and M.J. Schnitzer. 2004. In vivo mammalian brain imaging using one- and two-photon fluorescence microendoscopy. J. Neurophysiol. 92:3121-3133.

12. Levene, M.J., D.A. Dombeck, R.M. Williams, J. Skoch, G.A. Hickey, K.A. Kasischke, R.P. Molloy, M. Ingelsson, et al. 2004. In vivo multiphoton microscopy of deep tissue with gradient index lenses. Proceedings of SPIE, Photonics West. p. 291-296.

13. Kiesslich, R., J. Burg, M. Vieth, J. Gnaendiger, M. Enders, P. Delaney, A. Polglase, W. McLaren, et al. 2004. Confocal laser endoscopy for diagnosing intraepithelial neoplasias and colorectal cancer in vivo. Gastroenterology 127:706-713.

14. Kiesslich, R., M. Goetz, J. Burg, M. Stolte, E. Siegel, M. Maeurer, S. Thomas, D. Strand, et al. 2005. Diagnosing Helicobacter pylori in vivo by confocal laser endoscopy. Gastroenterology 128:2119-2123.

15. Lademann, J., N. Otberg, H. Richter, L. Meyer, H. Audrig, A. Teichmann, S. Thomas, A. Knuttel, and W. Sterry. 2007. Application of optical non-invasive methods in skin physiology: a comparison of laser scanning microscopy and optical coherent tomography with histological analysis. Skin Res. Technol. 13:119-132.

16.Swindle, L.D., S.G. Thomas, M. Freeman, and P.M. Delaney. 2003. View of normal human skin in vivo as observed using fluorescence fibre-optic confocal microscopic imaging. J. Invest. Dermatol. 121:706-712.

17. Wells, A.P., L. Wakely, W. Birchall, and P.M. Delaney. 2006. In vivo fiberoptic confocal imaging (FOCI) of the human ocular surface. J. Anat. 208:197-203.

18. Goetz, M., C. Fottner, E. Schirrmacher, P. Delaney, S. Gregor, C. Schnieder, D. Strand, S. Kanzler, et al. 2007. In-vivo confocal realtime mini-microscopy in animal models of human inflammatory and neoplastic diseases. Endoscopy 39:350-356.

19.Zipfel, W.R., R.M. Williams, and W.W. Webb. 2003. Nonlinear magic: multiphoton microscopy in the biosciences. Nat. Biotechnol. 21:1369-1377.

20. Helmchen, F., M.S. Fee, D.W. Tank, and W. Denk. 2001. A miniature head-mounted two-photon microscope: high resolution brain imaging in freely moving animals. Neuron 31:903-912.

21. Ouzounov, D.G., F.R. Ahmad, D. Muller, N. Venkataraman, M.T. Gallagher, M.G. Thomas, J. Silcox, K.W. Koch, and A.L. Gaeta. 2003. Generation of megawatt optical solitons in hollow-core photonic band-gap fibers. Science 301:1702-1704.

22.Ouzounov, D.G., K.D. Moll, M.A. Foster, W.R. Zipfel, W.W. Webb, and A.L. Gaeta. 2002. Delivery of nanojoule femtosecond pulses through large-core microstructure fiber. Opt. Lett. 27:1513-1515.

23. Ramachandran, S., M.F. Yan, J. Jasapara, P. Wisk, S. Ghalmi, E. Monberg, and F.V. Dimarcello. 2005. High energy (nanojoule) femtosecond pulse delivery with highly dispersive higher order mode fibers. Opt. Lett. 30:3225-3227.

Address correspondence to Simon C. Watkins, Departments of Environmental and Occupational Health and Cell Biology and Physiology, University of Pittsburgh, Pittsburgh, PA 15261, USA. e-mail: swatkins@pitt.edu

To purchase reprints of this article, contact: Reprints@BioTechniques.com 